\title{
Enhanced GPSR Routing in Multi-Hop Vehicular Communications through Movement Awareness
}

\author{
Fabrizio Granelli ${ }^{*}$, Giulia Boato ${ }^{*}$, Dzmitry Kliazovich ${ }^{*}$ and Gianni Vernazza ${ }^{\#}$ \\ * DIT - University of Trento \\ Via Sommarive 14, I-38050 Trento (ITALY) \\ E-mail: [granelli, boato, klezovic]@dit.unitn.it \\ \# DIBE - University of Genoa \\ Via Opera Pia 13, Genoa (ITALY) \\ E-mail: vernazza@dibe.unige.it
}

\begin{abstract}
Providing reliable and efficient routing in presence of relative movement motivates the introduction of movement awareness to improve performance of existing position-based routing schemes in vehicular ad-hoc networks. The proposed algorithm represents a modification of well-known GPSR which exploits information about movement in order to improve the next forwarding node decision. Performance evaluation of the proposed protocol underlines a promising and robust basis for designing a routing strategy suitable for the automotive scenario. ${ }^{1}$
\end{abstract}

Keywords: Routing, Vehicular Ad-Hoc Communications, VANET.

\section{INTRODUCTION}

A growing interest in the field of ICT for automotive applications lies in the possibility of enabling vehicles to communicate using wireless technology, in order to access the Internet or other network commodities, or more in general to support other services for the users. This feature is envisaged to be implemented into three ways: (i) by the deployment of proper communication infrastructure along the roads to act as gateways to the Internet (Vehicle-toInfrastructure Communication or V2I), (ii) by the implementation of the so-called "vehicle ad-hoc network" (Vehicle-to-Vehicle Communication, V2V or VANET in the multi-hop case), or (iii) by a combination of the two: a multihop wireless network built by vehicles and fixed gateways. In this framework, guidelines for providing vehicle-tovehicle communications as well as a reference protocol architecture are being proposed by the Car-2-Car Communication Consortium [1], leaving the floor to further study and proposals especially in the context of routing.

Indeed, the basic concept of VANETs derives from the well-known model of the mobile ad-hoc networks (MANETs), infrastructure-less networks where wireless hosts communicate with each other in the absence of a fixed infrastructure. Multihop data communication in VANETs is usually achieved via location-based ad hoc routing protocols, a class of multihop routing for ad hoc networks [2]. A relevant analysis of perspective services of vehicle networking and their mapping into requirement on the networking infrastructure is presented by Bai et al. in [3],

${ }^{1}$ This work is partially funded by EU in the framework of SAFESPOT Integrated Project (VI F.P.). where it is clearly outlined that several services (ranging from safety to convenience) require multi-hop communications, and specifically "one-to-one" (i.e. a single end-to-end path) and "one-to-zone" (i.e. route "till an area and then local geo-referenced broadcast) routing strategies.

This paper illustrates how a candidate routing algorithm for VANETs (Greedy Perimeter Stateless Routing [4]) can benefit from the introduction of movement awareness in a VANET scenario. The proposed algorithm, GPSR-MA (Greedy Perimeter Stateless Routing with Movement Awareness), exploits not only the position, but also the direction and speed of movement of mobile nodes. GPSRMA, by taking advantage of information about vehicle movement, represents an attempt to find a solution for links stability and therefore improve routing robustness - matching the requirements of both $\mathrm{V} 2 \mathrm{~V}$ and V2I communication.

The structure of the work is the following: Section II presents an overview of GPSR and the modifications introduced by GPSR-MA. Evaluation results obtained in a highway and plane grid scenarios are discussed in Section III, while final remarks are presented in Section IV.

\section{GPSR AND GPSR-MA}

\section{A. GPSR Overview}

Location based greedy forwarding routing is one of the most promising routing approaches for VANETs: in Greedy Perimeter Stateless Routing (GPSR) [4], all packets transmitted onto the network are marked by the originator with their destination's locations. Assuming to know the exact positions of their neighbors, GPSR nodes choose to forward the packet to the neighbor located closer to the destination. If greedy forwarding is not possible due to the network topology, GPSR recovers by traversing the topology graph around the perimeter of this region. However, since in GPSR only position information is used, it may be possible to lose some good candidates to forward the packet, in particular in a network where nodes are highly mobile, such as a VANET. In this scenario, it is important to guarantee high stability of the links and the use of node's position only could not be sufficient.

GPSR specifies a simple beaconing algorithm used by network nodes (i.e. the vehicles) to update their neighbors with positioning information at regular intervals $\mathrm{B}$. The value of $B$ represents a tradeoff between routing overhead and level of mobility in the network, and thus it should be carefully tuned to the considered scenario. With the realistic 
assumption of a car speed of $100 \mathrm{~km} / \mathrm{h}$ and $\mathrm{B}=1 \mathrm{sec}$, the location can be changed of up to 30 meters between updates. This is already a considerable fraction of a node's transmission range, which can limit GPSR performance in terms of accuracy in neighbor selection.

A bigger problem is related to the accuracy of destination positioning. In GPSR, it is assumed the packet source can determine the coordinates of the destination node using a location lookup service: each node registers itself with a location server performing subsequent positioning updates. A source node having a data ready to send should (i) obtain destination coordinates of the destination, and then (ii) perform periodic location updates with queries to the lookup service. In networks with no location lookup service available, destination coordinates can be obtained with a traditional destination lookup flooding. As a result, location lookup procedure requires considerable amount of time and network resources in order to maintain highly accurate destination coordinates in case of high mobility of network nodes. In our study (presented in Section III), positioning error can go far beyond transmission range of the node immediately resulting in drop of in-transit packets due to route failure, as routing is performed in a region where the destination node is not present any more. This situation can be recovered only after destination coordinates are updated within the location lookup service.

Summarizing, beaconing interval B and location update interval $Q$ are the key parameters defining GPSR performance, as such parameters define the accuracy of route selections and influence routing protocol overhead. In presence of high mobility, like in case of vehicles moving on a highway, B and Q should be relatively small in order to avoid frequent route breaks due to inaccurate position estimation.

Since movement is the primary reason for inaccuracy of position based routing protocols, we consider including it into a proper routing metric. In case of GPSR this can be considered as a routing metric extension exploiting also the first derivative of the position to account for frequent changes of coordinate function. This will enable relevant advantages: a more accurate and stable routing procedure and a reduced routing overhead - thus releasing network resources.

\section{B. GPRS with Movement Awareness (GPSR-MA)}

The mobility aware extension of GPSR protocol, called Greedy Perimeter Stateless Routing with Movement Awareness (GPSR-MA), extends the set of parameters used for taking a routing decision with the inclusion of (i) the speed and (ii) the direction of movement of the vehicle.

Speed is an absolute value measured in $\mathrm{m} / \mathrm{s}$, while movement direction is an absolute angle between node's speed vector and the segment connecting it to the destination. Nodes include such parameters into periodic location update packets, which in our implementation corresponds to an overhead of two octets added to the GPSR header. Alternatively, speed and direction may not be reported explicitly but can be derived by neighboring nodes using a history of node's coordinates.

It should be underlined that GPSR-MA functionality is kept fully consistent with the original GPSR specification - including the "stateless" property where every node relies only on local state information associated with its direct neighbors. GPSR-MA enhancements are the following:

Position Prediction: As soon as movement direction and speed become available, every node is able to instantaneously update location coordinates of its neighbors without the need for communication. To this aim, each node is provided with a high resolution timer (at msec. level) upon expiration of which it traverses the neighbor node table adjusting neighbors' positions accounting for the range they moved since the last position update beacon was received from them. In addition, the destination coordinates carried inside every transmitted data packet are adjusted accordingly.

Assuming B and Q intervals implemented with a $1 \mathrm{sec}$. resolution timers, a node (a car) is not likely to change much its speed and movement direction between beacon updates. As a result, the adjusted positioning information of the neighbors will be highly accurate.

Furthermore, in order to ensure up-to-date coordinates registered in the location lookup service, the destination node computes the positioning error by checking destination coordinates in every data packet inserted by the source node. In case the difference of the received (destination) coordinates with real ones is above a predefined threshold, the destination proactively issues position update message to the location lookup service. This threshold can be set as a fraction of the transmission range or represent a predefined parameter. From evaluation experiments (presented in Section III), $1 / 4^{\text {th }}$ of the transmission range seems to be an adequate value.

Routing Metric: In order to increase path robustness the proposed routing metric favors relatively stable paths. To this aim, we define a metric which depends on i) speed, ii) distance from the destination, and iii) movement direction as follows:

$$
\begin{aligned}
& m(s, d, \theta)=\alpha_{\text {speed }} f(s)+\alpha_{\text {distance }} g(d)+\alpha_{\text {movement }} h(\theta) \\
& \text { where } \alpha_{\text {speed }}, \alpha_{\text {distance }}, \alpha_{\text {movement }} \text { are different weights }
\end{aligned}
$$
associated to speed, distance and movement direction and $f(s), g(d), h(\theta)$ are the speed, distance and movement weighting functions, respectively.

The factor related to speed is defined to favor the next forwarding node moving with a speed close to the speed of current forwarder:

$$
f(s)=\exp \left(-\left(x-s_{i}\right)^{2} / 2 \sigma^{2}\right)
$$

where $s_{i}$ is the reference speed of current node $\mathrm{i}$.

The distance component (as in GPSR) chooses forwarding nodes located closer to the destination, but considering that the next hop must be within the transmission range and preferably not to close to its perimeter (therefore excluding nodes closer than a tolerance factor):

$$
g(d)=\exp (-(d-l))
$$

where $l=d_{i}-$ transmission range + tolerance, $d_{i}$ is the distance of current node $i$ from destination and tolerance is a parameter which will depend on the scenario: the higher the speed of nodes the higher is its value. 
Focusing on movement direction, GPSR-MA favors nodes moving towards the destination (with an angle $\theta=0$ ):

$$
h(\theta)=\left\{\begin{array}{cc}
0 & \text { if } \theta \geq \frac{\pi}{2} \\
\cos ^{2}(\theta) & \text { elsewhere }
\end{array}\right.
$$

where $\theta$ is an angle between node's movement direction and the line connecting it to the destination.

\section{PERFORMANCE EVALUATION}

Comparison is performed against original GPSR protocol. Performance comparison of GPSR with the state of the art protocols such as DSR and AODV can be found in [4]. Simulations are performed using $n s-2$ simulator with $\alpha_{\text {speed }}=\alpha_{\text {distance }}=\alpha_{\text {movement }}=1 / 3$.

Highway scenario: On a two-lane highway four cars are moving on the right lane and $n$ cars on the left lane. The distance between neighboring cars is chosen to be 70 meters, while the transmission range is fixed to 100 meters. All vehicles maintain a fixed speed in the range from 0 to $30 \mathrm{~m} / \mathrm{s}$ $(108 \mathrm{~km} / \mathrm{h})$. Duration of the experiment is limited to 1 minute.

Fig. 1 presents positioning error of destination node in GPSR, which shows that even for frequent position updates the positioning error is a considerable fraction of node's transmission range - highlighting the need for introducing movement awareness.

Fraction of application packets delivered (Fig. 2 and Fig. 3) and TCP throughput against movement speed (Fig. 4) demonstrate robustness of GPSR-MA as well as insensitiveness to position update intervals which allows to maintain high performance with minimal routing overhead.
In fact, hello and location query intervals can be set to the highest value while maintaining highest performance, thus reducing signaling overhead.

Plane grid: 60 nodes are randomly distributed in $1500 \times 300$ meters area, and move with random waypoint model $(\max$ speed $=25 \mathrm{~m} / \mathrm{s})$. A single CBR UDP connection is set between randomly chosen source and destination nodes. Results are averaged over 10 runs. Achieved results (Fig. 5) outline the enhancement deriving from the implementation of movement awareness in GPSR.

\section{CONCLUSIONS}

The paper proposes a modified routing scheme for VANETs, GPSR-MA, which represents an extension of well-known GPSR - enabling it to exploit information about movement in order to improve the next forwarding node decision. Extensive simulations evaluating the proposed protocol demonstrate that GPSR-MA provides a promising and robust basis for designing a routing strategy suitable for the automotive scenario.

\section{REFERENCES}

[1] "Car2Car Communication Consortium. Conformance Guideline for Projects and Demonstrators". Preliminary Version 0.1.

[2] J. Chennikara-Varghese, W. Chen, O. Altintas and S. Cai. "Survey of routing Protocols for Inter-Vehicle Communications." Proc. of Vehicle-to-Vehicle Communications Workshop 2006, Jul. 2006.

[3] F. Bai et al. "Towards Characterizing and Classifying Communication-based Automotive Applications from a Wireless Networking Perspective." Proc. of IEEE Workshop on Automotive Networking 2006, Dec. 2006.

[4] B. Karp and H. T. Kung. "GPSR: Greedy Perimeter Stateless Routing for Wireless Networks," Proc. of ACM/IEEE International Conference on Mobile Computing and Networking 2000, Aug. 2000.

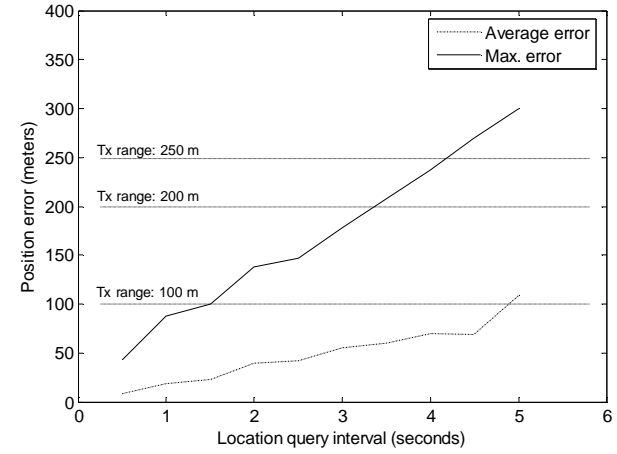

Figure 1. GPSR error in position estimation. (speed: $30 \mathrm{~m} / \mathrm{s}$, hello interval $=1 \mathrm{sec}$.)

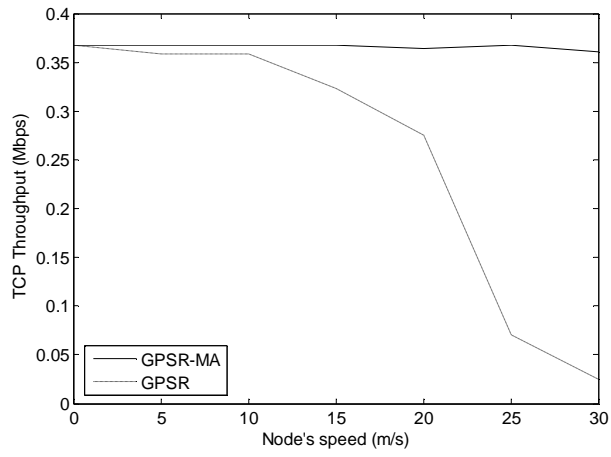

Figure 4. TCP throughput against movement speed.

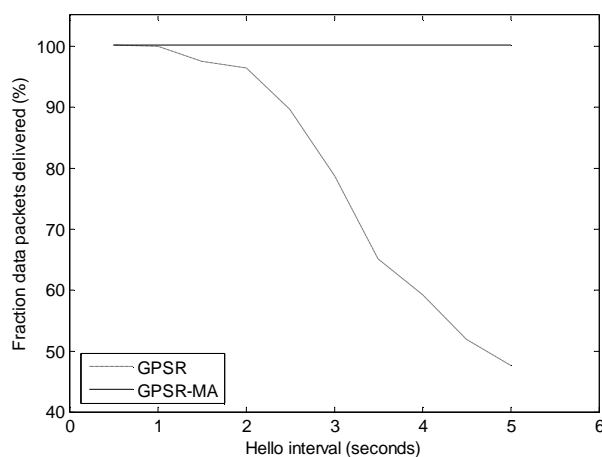

Figure 2. Packet delivery ratio against hello message interval

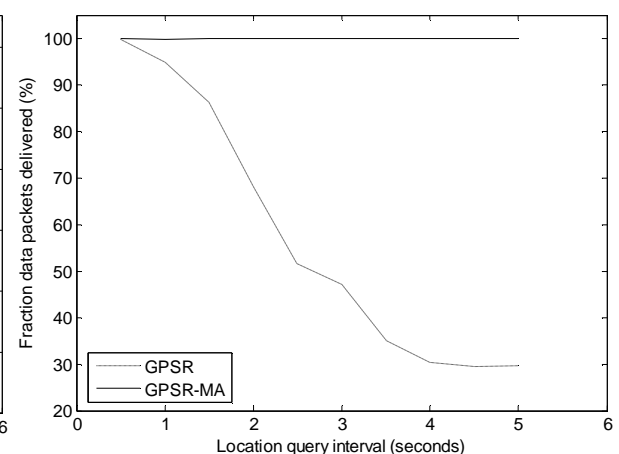

Figure 3. . Packet delivery ratio against location query interval

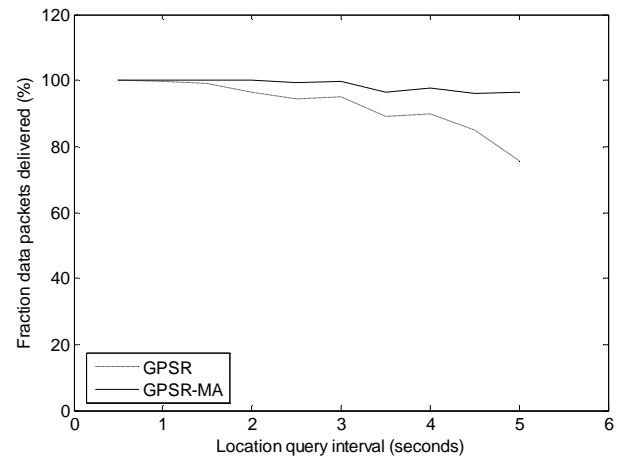

Figure 5. Application packet delivery ratio in GPSR and GPSR-MA. 